\title{
Review: small treatment effect of disodium cromoglycate in childhood asthma is tempered by methodological flaws and publication bias
}

\author{
Tasche MJ, Uijen JH, Bernsen RM, et al. Inhaled disodium cromoglycate (DSCG) as maintenance therapy in children with \\ asthma: a systematic review. Thorax 2000 Nov;55:913-20.

\section{QUESTION: In children with asthma, how effective is inhaled disodium cromoglycate (DSCG)?}

\section{Data sources}

Studies were identified by searching Medline, EMBASE/ Excerpta Medica, the Cochrane Controlled Trials Register, and the database of the manufacturers of DSCG with the terms asthma, sodium cromoglycate, and clinical trial. Bibliographies of relevant studies were reviewed.

\section{Study selection}

English language studies were selected if they were randomised, double blind, placebo controlled trials of inhaled DSCG in the maintenance treatment of asthma in children up to 18 years of age. Studies of exercise induced asthma were excluded.

\section{Data extraction}

Data were extracted on methodological quality, setting, design, DSCG dose and method of administration, patient characteristics, duration of intervention, outcomes, and adverse effects. Symptom scores were calculated separately for wheeze and cough. Pooled estimates of the treatment effect were calculated by using random effects models for absolute and relative effects.

\section{Main results}

24 trials were included, and patient numbers ranged from 9 to 218. All children had moderate to severe asthma, and the median duration of intervention was 4 weeks. Study results favoured DSCG in 16 trials, were partially positive in 3 , and were equivalent in 5 . Heterogeneity was present among the trials. The absolute treatment effects for cough and wheeze on a 0 to 3 scale were statistically significant in favor of DSCG (95\% CI 0.11 to 0.26 and CI 0.13 to 0.26 , respectively). The relative improvement in means (the difference in mean score divided by the placebo score) for cough and wheeze was also significant (CI for the relative improvement in mean 0.16 to 0.37 and CI 0.19 to 0.36 , respectively). Funnel plots showed significant publication bias by the absence of small, negative trials $(p=0.095$ for cough and $p=0.01$ for wheeze).

\section{Conclusions}

In children with asthma, a meta-analysis of 24 randomised controlled trials shows a small treatment effect of inhaled disodium cromoglycate. The results are tempered by mediocre methodological quality of the trials, heterogeneity, and publication bias.

\section{COMMENTARY}

The systematic review by Tasche $e t$ al had a well focused question, and the criteria used to select articles for inclusion were appropriate. The authors point out that important relevant studies may have been missed (examination of the funnel plot) but that their exclusion would further dilute, not enhance, the effect of DSCG. Acknowledging that the methodological quality of the trials was mediocre, the authors statistically pooled the results and showed that the tolerance interval included zero. They concluded that it is no longer justified to use DSCG as a firstline prophylactic agent in childhood asthma.

This meta-analysis, together with the results of the following studies,${ }^{12}$ may encourage many to use inhaled corticosteroids (ICSs) as firstline treatment. One trial showed that in children with mild to moderate asthma, inhaled budesonide improved airway responsiveness and provided better control of asthma than placebo or nedocromil. Its side effects were limited to a small, transient reduction in growth velocity. ${ }^{1}$ Another trial concluded that children with asthma who received long term treatment with budesonide attained normal adult height. ${ }^{2}$

Because asthma is the most common chronic disease in childhood, the clinical application of this evidence is important. If the concerns of ICSs on growth are no longer an issue, some may think that ICSs are as safe as other anti-inflammatory agents. However, the long term effects of steroids on the developing immune system are still unknown. Until long term marketing surveillance establishes the safety of ICSs, clinicians must continue to weigh the risks and benefits when choosing ICSs over DSCG.

Lynnette J Mazur, MD, MPH University of Texas Houston, Texas, USA

1 The Childhood Asthma Management Program Research Group. Long-term effects of budesonide or nedocromil in children with asthma. N Engl J Med 2000;343:1054-63.

2 Agertoft L, Pedersen S. Effect of long-term treatment with inhaled budesonide on adult height in children with asthma. N Engl J Med 2000;343:1064-9. 